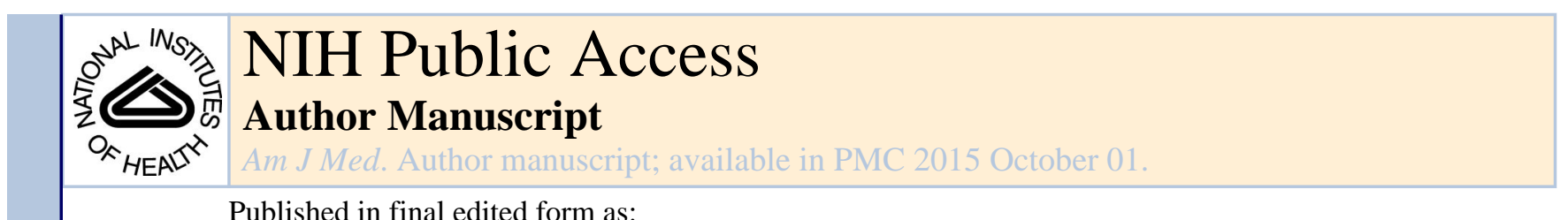

Published in final edited form as:

Am J Med. 2014 October ; 127(10): 1012-1016. doi:10.1016/j.amjmed.2014.05.004.

\title{
Long-term Outcomes of Enhanced Depression Treatment in Patients with Acute Coronary Syndromes
}

\author{
Siqin Ye, MD, MS ${ }^{a}{ }^{*}$, Jonathan A. Shaffer, $\mathrm{PhD}^{\mathrm{a}}$, Nina Rieckmann, $\mathrm{PhD}^{\mathrm{b}}$, Joseph E. \\ Schwartz, PhD ${ }^{\mathrm{a}, \mathrm{c}}$, lan M. Kronish, MD, MPH ${ }^{\mathrm{a}}$, Joseph A. Ladapo, MD, PhD ${ }^{\mathrm{d}}$, William Whang, \\ MD $^{\mathrm{a}}$, Matthew M. Burg, $\mathrm{PhD}^{\mathrm{a}, \mathrm{e}}$, and Karina W. Davidson, $\mathbf{P h D}^{\mathrm{a}}$ \\ ${ }^{a}$ Center for Behavioral Cardiovascular Health, Department of Medicine, Columbia University \\ College of Physician \& Surgeons, New York, New York \\ ${ }^{b}$ Berlin School of Public Health, Charité Universitätsmedizin, Berlin, Germany \\ 'Department of Psychiatry and Behavioral Sciences, SUNY-Stony Brook University, Stony Brook, \\ New York \\ dDepartment of Population Health, New York University School of Medicine, New York, New York \\ eDepartment of Internal Medicine, Yale University School of Medicine, New Haven, Connecticut
}

\section{Abstract}

Background-The Coronary Psychosocial Evaluation Studies (COPES) trial demonstrated promising results for enhanced depression treatment to reduce cardiovascular risk of patients with acute coronary syndrome and comorbid depression, but the long-term effectiveness of this intervention is unclear.

\begin{abstract}
Methods-157 participants with persistent depression after hospitalization for acute coronary syndromes were enrolled in the COPES trial. 80 participants were allocated to 6-months of enhanced depression treatment, and 77 were allocated to usual care. We report on an additional 12 months of observational follow-up for the composite outcome of death or first hospitalization for myocardial infarction or unstable angina.
\end{abstract}

Results-Although the intervention was previously shown to have favorable cardiovascular effects during the treatment period, we observed a significant time-by-treatment group interaction during extended follow-up ( $\mathrm{p}=0.008$ ). Specifically, during the 6-month treatment period, death or hospitalization for myocardial infarction / unstable angina occurred in 3 (4\%) participants in the

(C) 2014 Elsevier Inc. All rights reserved.

*Correspondence to: Siqin Ye, MD, MS Center for Behavioral Cardiovascular Health Columbia University Medical Center 622 West 168th Street, PH 9-320 New York, NY 10032 sy2357@ columbia.edu Phone: 212-305-5633 Fax: 212-342-3431.

Publisher's Disclaimer: This is a PDF file of an unedited manuscript that has been accepted for publication. As a service to our customers we are providing this early version of the manuscript. The manuscript will undergo copyediting, typesetting, and review of the resulting proof before it is published in its final citable form. Please note that during the production process errors may be discovered which could affect the content, and all legal disclaimers that apply to the journal pertain.

Clinical Trial Registration: clinicaltrials.gov identifier NCT00158054 (http://clinicaltrials.gov/show/NCT00158054)

Conflicts of Interest: The authors report no additional conflicts of interest.

All authors had access to the data and contributed to writing of the manuscript. This work was presented, in part, as an abstract at the American College of Cardiology Scientific Sessions 2013. 
treatment group, compared with $11(14 \%)$ in the usual care group $(\mathrm{HR}=0.25,95 \%$ confidence interval [CI $]$ 0.07-0.90, $\mathrm{p}=0.03$ ). In contrast, during 12 months of additional observational followup, $11(14 \%)$ participants in the treatment group experienced the composite outcome, as compared to $3(4 \%)$ in the usual care group $(\mathrm{HR}=2.91,95 \%$ CI $0.80-10.56, \mathrm{p}=0.10)$.

Conclusions-Enhanced depression treatment was associated with a reduced risk of death or hospitalization for myocardial infarction / unstable angina during active treatment, but this effect did not persist after treatment ceased. Future research is needed to confirm our findings, and to determine the optimal duration of depression treatment in patients with depression after acute coronary syndromes.

\section{Keywords}

depression; acute coronary syndrome

\section{Introduction}

Depression is associated with increased risk for death and major adverse cardiac events in patients with coronary heart disease (1). Prior trials of brief depression treatment have failed to demonstrate long-term benefits on cardiovascular outcomes $(2,3)$. In the Coronary Psychosocial Evaluation Studies (COPES) randomized controlled trial, we have previously reported that patient-preference based, enhanced depression treatment compared to usual care in patients with acute coronary syndromes may reduce the risk of death or major adverse cardiac events during the 6-months treatment period (4). To address whether this treatment effect was durable when active treatment ended, we examined cardiac events and mortality in the COPES trial through an additional 12 months of observational follow-up after the end of the 6-month treatment period.

\section{Methods}

As previously described (4), 157 patients with a score of $\geq 10$ on the Beck Depression Inventory (5) within 1 week of acute coronary syndrome hospitalization and again at 3month follow-up were recruited from January 1, 2005, through February 29, 2008. Patients were randomized to either usual care or to enhanced depression treatment involving stepped, patient-preference driven care with problem-solving therapy, pharmacotherapy, or both. Demographic data and medical history were collected at index hospitalization. During the 6month treatment period and for an additional 12 months after treatment ended, hospitalization information was collected for all patients, and hospital systems were surveyed actively for hospitalizations of trial participants. Hospitalization records were adjudicated in a blinded fashion by two cardiologists to determine occurrences of major adverse cardiac events, defined as nonfatal myocardial infarction or hospitalization for unstable angina; a third cardiologist resolved disagreements. The National Death Index was searched to confirm reported deaths and to determine vital status of those who could not be contacted.

For this analysis, we constructed Cox proportional hazards models to estimate the effect of enhanced depression treatment on time-to-death or first major adverse cardiac event, 
adjusted for age, sex, Global Registry of Acute Coronary Events risk score (6), and left ventricular ejection fraction. Because a formal significance test based on scaled and unscaled Schoenfeld residuals confirmed that the treatment effect differed between the treatment and the observational follow-up time periods $(\mathrm{p}=0.008),(7)$ treatment assignment was entered in the model as a time-varying covariate, with separate estimates reported for the effect of enhanced depression treatment during the 6-month intervention period and for the 12-month subsequent observational follow-up.

\section{Results}

Of the 157 participants with persistent depressive symptoms 3 months after an index acute coronary syndrome hospitalization, 80 were randomized to enhanced depression treatment and 77 were randomized to usual care (Figure 1), as has been reported previously.(4) There was no significant difference between the two groups with regards to age, sex, race, ethnicity, education level, marital status, baseline Beck Depression Inventory score, Global Registry of Acute Coronary Events risk score, revascularization status during index hospitalization, or left ventricular ejection fraction (Table 1).

The median follow-up period was 11.8 months after treatment ended. During the 6-month intervention period, $11(14 \%)$ participants in the usual care arm experienced the composite outcome of death or hospitalization for myocardial infarction / unstable angina, including one participant whose death was not identified in our previously published analysis.(4) In comparison, 3 (4\%) participants experienced the composite outcome in the intervention arm during the intervention period (HR=0.25, 95\% confidence interval $[\mathrm{CI}] 0.07-0.90, \mathrm{p}=0.03$ ). At the beginning of the 12-month observational follow-up period, 58 of 77 (75\%) patients in the control group had Beck Depression Inventory score of $\geq 10$, compared to 46 of 80 (58\%) patients in the intervention group $(\mathrm{p}=0.018)$, suggesting that a substantial number of participants continued to have depressive symptoms. During this time period, 3 (4\%) participants in the usual care arm experienced the composite outcome of death or hospitalization for myocardial infarction / unstable angina, while $11(14 \%)$ participants in the intervention arm had the composite outcome $(\mathrm{HR}=2.91,95 \%$ CI $0.80-10.56, \mathrm{p}=0.10)$ (Table 2 and Figure 2).

\section{Discussion}

During the observational follow-up of the COPES randomized controlled trial, we found that the original finding of a reduced risk of death or hospitalization for myocardial infarction / unstable angina during the 6-month treatment period did not persist after treatment ended. Furthermore, there was a significant time-treatment interaction in the opposite direction, suggesting that there may have been a "catch-up" in cardiovascular events after the intervention ended. To our knowledge, this is the first report of a "catch-up" phenomenon concerning cardiac events after the end of depression treatment, with implications for future trials of depression management in patients with coronary heart disease.

Recent trials of novel depression interventions in this patient population have mainly examined the effect of interventions during the period in which depression treatment is 
offered. In a trial of 214 participants from 14 primary care clinics, Katon and colleagues showed that a stepped, patient-preference based intervention led to improvements in both depressive symptoms and management of coronary heart disease, compared to usual care, during the 12-months treatment period.(8) Similarly, Blumenthal and colleagues recently reported that for patients with coronary heart disease and elevated depressive symptoms, a 4-month structured exercise intervention improved heart rate variability, a marker of cardiac risk, as compared to those receiving sertraline or placebo, at the end of the 4-month period. (9) However, as the current findings suggest, the extent to which the benefits reported in these studies will persist after the completion of the interventions tested cannot be assumed. Future trials targeting depression in coronary heart disease patients may need to consider longer treatment duration, as well as evaluate the benefits and costs of ongoing symptom monitoring and "booster" treatments.

There are several potential mechanisms for the "catch up" phenomenon in cardiac risk that we observed. Depression is associated with biological processes, such as inflammation and endothelial dysfunction,(10-12) and behavioral processes, such as smoking and physical inactivity,(13) that are known to contribute to worse outcomes after hospitalizations for acute coronary syndromes. A 6-month course of depression treatment may not be sufficient to permanently affect these pathways, or may be too brief to prevent a rebound in these processes after treatment discontinuation. Alternatively, since depression is a chronic illness defined by a remitting-relapsing course, it is possible that patients with untreated or undertreated relapses in depressive symptoms post-intervention may have particularly high cardiovascular risk during those time-periods of symptom rebound. A better understanding of the complex interactions between depression treatment, depressive symptoms, and the biological and behavioral mediators of cardiac risk in patients with depression after an acute coronary syndrome event is needed to better understand the "catch-up" phenomenon for cardiac risk that we observed.

The current findings have several limitations, including the small study size and the post-hoc nature of the analyses. Given the small number of events, it is possible that chance finding or regression to the mean could explain the "catch-up" phenomenon we observed. In addition, differential loss to follow-up due to a healthy survivor effect cannot be ruled out. It is also unknown whether participants undertook cardiac rehabilitation or other activities that could affect quality of life and cardiac risk during the treatment and follow-up time periods. Furthermore, it is unknown what types of depression treatment, if any, were received by participants during the observational follow-up. These findings are thus hypothesisgenerating and require replication. Nonetheless, our study raises the possibility that the treatment of depression in patients with acute coronary syndromes may need to be of longer duration to maintain the cardiac benefits observed during treatment. Future research is needed to confirm our findings, to determine the optimal duration of initial depression treatment in this patient population, and to identify treatment approaches that will deliver durable results. 


\section{Acknowledgments}

Funding Sources: This COPES study was supported by National Heart, Lung, and Blood Institute grants HC-25197, and is registered at clinicaltrials.gov (clinicaltrials.gov identifier NCT00158054, http://clinicaltrials.gov/ show/NCT00158054).

\section{References}

1. Carney RM, Freedland KE. Depression in patients with coronary heart disease. Am J Med. 2008; 121(11 Suppl 2):S20-7. [PubMed: 18954589]

2. Berkman LF, Blumenthal J, Burg M, et al. Effects of treating depression and low perceived social support on clinical events after myocardial infarction: the Enhancing Recovery in Coronary Heart Disease Patients (ENRICHD) Randomized Trial. JAMA. 2003; 289(23):3106-16. [PubMed: 12813116]

3. Baumeister H, Hutter N, Bengel J. Psychological and pharmacological interventions for depression in patients with coronary artery disease. Cochrane Database Syst Rev. 2011; 9:CD008012. [PubMed: 21901717]

4. Davidson KW, Rieckmann N, Clemow L, et al. Enhanced depression care for patients with acute coronary syndrome and persistent depressive symptoms: coronary psychosocial evaluation studies randomized controlled trial. Arch Intern Med. 2010; 170(7):600-8. [PubMed: 20386003]

5. Beck AT, Ward CH, Mendelson M, et al. An inventory for measuring depression. Arch Gen Psychiatry. 1961; 4:561-71. [PubMed: 13688369]

6. Eagle KA, Lim MJ, Dabbous OH, et al. A validated prediction model for all forms of acute coronary syndrome: estimating the risk of 6-month postdischarge death in an international registry. JAMA. 2004; 291(22):2727-33. [PubMed: 15187054]

7. Grambsch P, Therneau T. Proportional hazards tests and diagnostics based on weighted residuals. Biometrika. 1994; 81:515-26.

8. Katon WJ, Lin EH, Von Korff M, et al. Collaborative care for patients with depression and chronic illnesses. N Engl J Med. 2010; 363(27):2611-20. [PubMed: 21190455]

9. Blumenthal JA, Sherwood A, Babyak MA, et al. Exercise and Pharmacological Treatment of Depressive Symptoms in Patients With Coronary Heart Disease: Results From the UPBEAT (Understanding the Prognostic Benefits of Exercise and Antidepressant Therapy) Study. J Am Coll Cardiol. 2012

10. Davidson KW, Schwartz JE, Kirkland SA, et al. Relation of inflammation to depression and incident coronary heart disease (from the Canadian Nova Scotia Health Survey [NSHS95] Prospective Population Study). Am J Cardiol. 2009; 103(6):755-61. [PubMed: 19268727]

11. Cooper DC, Tomfohr LM, Milic MS, et al. Depressed Mood and Flow-Mediated Dilation: A Systematic Review and Meta-Analysis. Psychosomatic Medicine. 2011; 73(5):360-9. [PubMed: 21636660]

12. Ye S, Denton E-g, Wasson L, Davidson K. Epidemiology and Management of Depression Following Coronary Heart Disease Diagnosis in Women. Current Cardiovascular Risk Reports. : $1-9$.

13. Ye S, Muntner P, Shimbo D, et al. Behavioral mechanisms, elevated depressive symptoms, and the risk for myocardial infarction or death in individuals with coronary heart disease: the REGARDS (Reason for Geographic and Racial Differences in Stroke) study. J Am Coll Cardiol. 2013; 61(6): 622-30. [PubMed: 23290548] 


\section{Clinical Significance}

- In the COPES trial, enhanced, patient-preference driven depression treatment reduced cardiovascular events for patients with acute coronary syndromes and comorbid depression during the 6-month treatment period

- During an additional 12 months of follow-up, we found that there was a rebound in cardiovascular risk after treatment ended

- These results indicate a need to determine optimal duration of depression treatment for patients with cardiovascular diseases and depression 


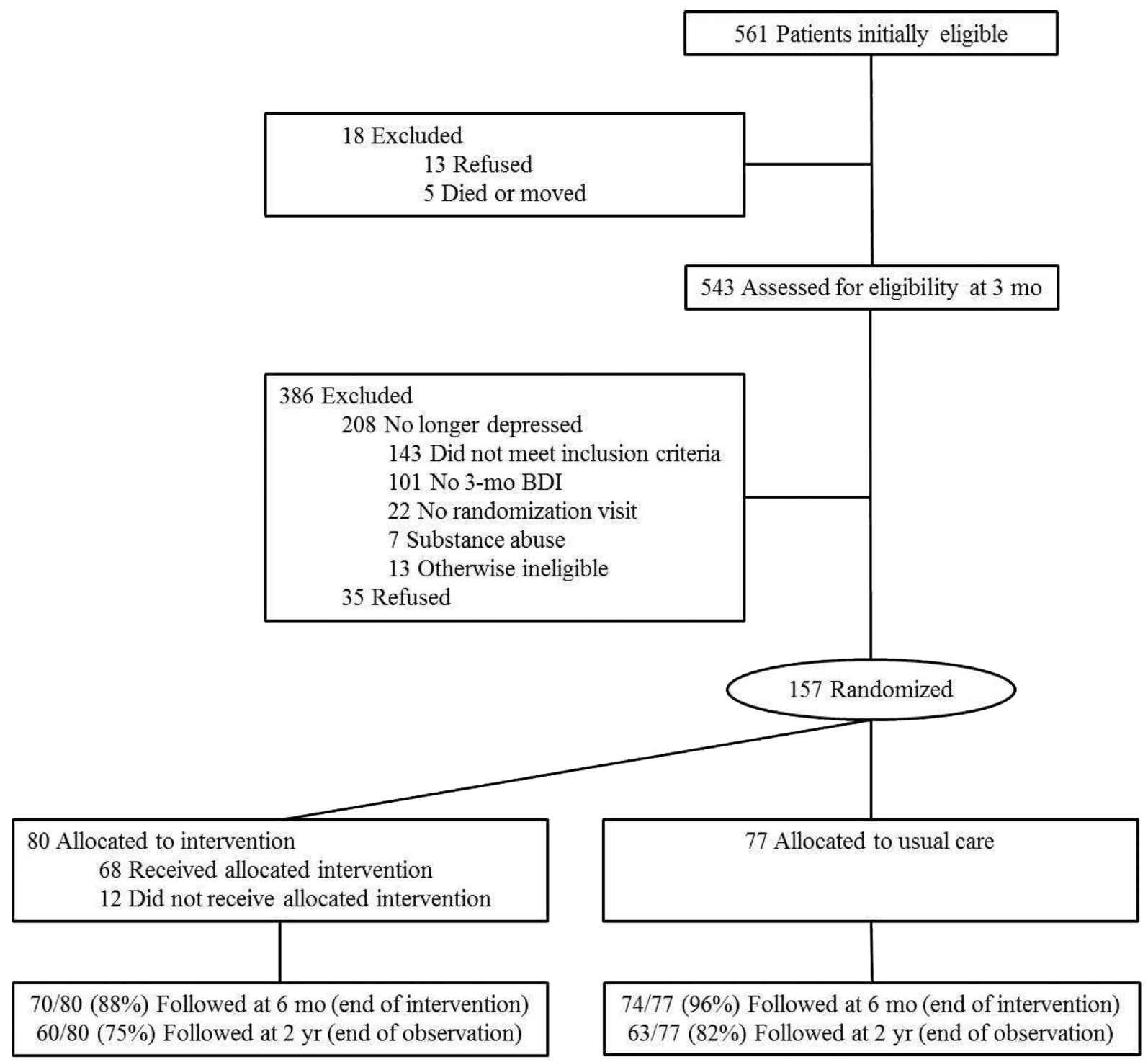

Figure 1.

Title: Patient flowchart. BDI indicates Beck Depression Inventory. 


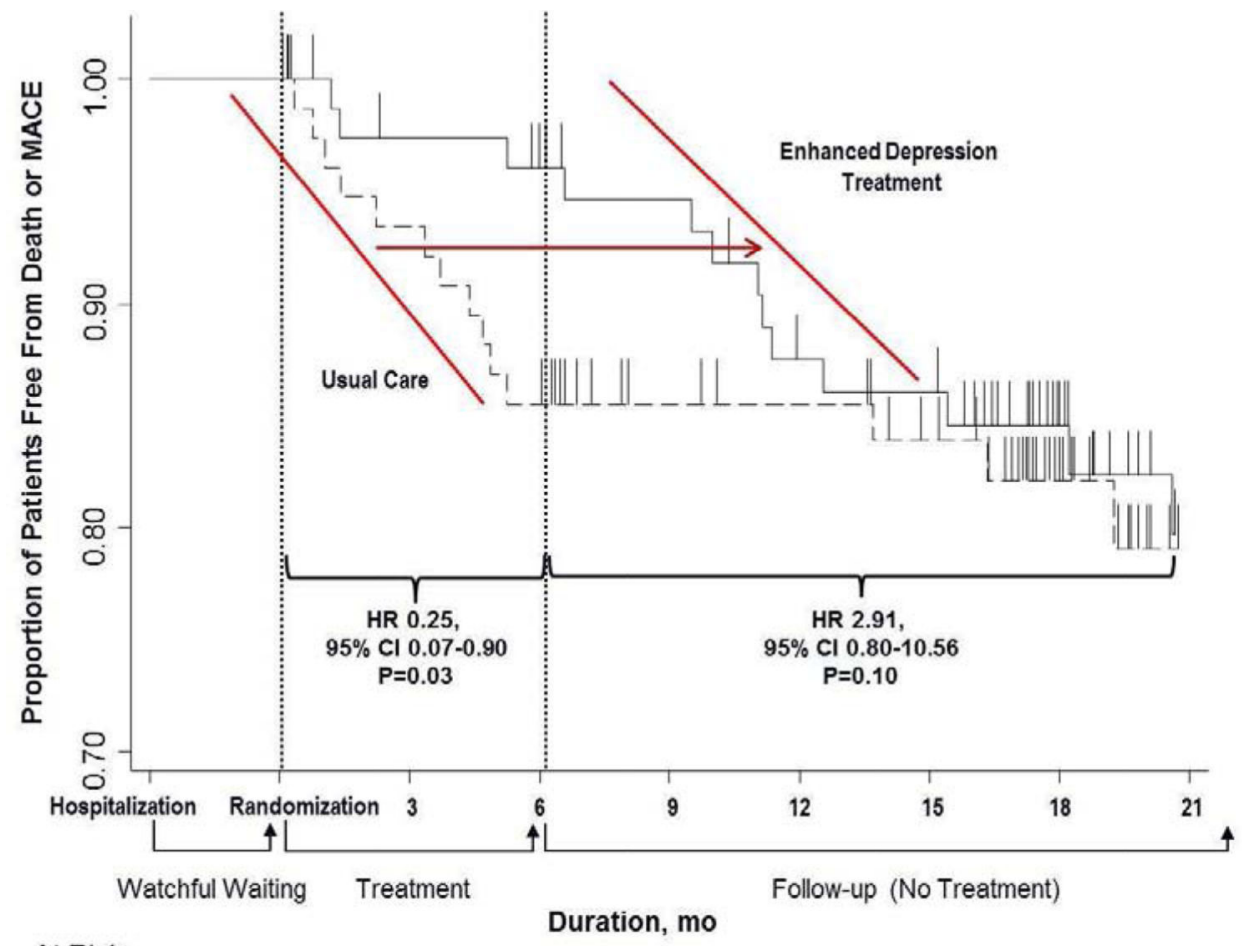

At Risk Usual Care Intervention

$\begin{array}{lll}77 & 71 & 64 \\ 80 & 73 & 70\end{array}$

56

56

54

49

33

18

Figure 2.

Title: Survival free from death or hospitalization for myocardial infarction or unstable angina, by treatment assignment. 


\section{Table 1}

Baseline characteristics of participants in the Coronary Psychosocial Evaluation Studies randomized, controlled trial, by treatment assignment.

\begin{tabular}{|c|c|c|c|}
\hline & Usual Care Group (n=77) & Intervention Group $(\mathbf{n}=\mathbf{8 0})$ & $P$ value \\
\hline Female, $n(\%)$ & $41(53)$ & $43(54)$ & 0.95 \\
\hline Age, years (SD) & $61.1(10.6)$ & $59.3(10.6)$ & 0.29 \\
\hline African American, n (\%) & $17(22)$ & $12(15)$ & 0.25 \\
\hline Hispanic, n (\%) & $32(42)$ & $36(45)$ & 0.66 \\
\hline Education (>= high school), n (\%) & $53(73)$ & $52(67)$ & 0.43 \\
\hline $\begin{array}{l}\text { Marital status, } \mathbf{n}(\%) \\
\text { Single } \\
\text { Married/partner } \\
\text { Separated / divorced / widowed }\end{array}$ & $\begin{array}{l}12(16) \\
36(48) \\
27(36)\end{array}$ & $\begin{array}{l}18(23) \\
34(44) \\
26(33)\end{array}$ & 0.54 \\
\hline $\mathrm{BDI}>=16$ at randomization, $\mathrm{n}(\%)$ & $52(68)$ & $52(65)$ & 0.74 \\
\hline Percutaneous coronary intervention at enrollment, $n(\%)$ & $66(86)$ & $62(78)$ & 0.19 \\
\hline Coronary artery bypass grafting at enrollment, $\mathbf{n}(\%)$ & $2(3)$ & $4(5)$ & 0.68 \\
\hline Globe Registry of Acute Coronary Events score, mean (SD) & $94(23)$ & $92(23)$ & 0.58 \\
\hline Left Ventricular Ejection Fraction $<40 \%$, n (\%) & $13(17)$ & $17(21)$ & 0.49 \\
\hline
\end{tabular}

Abbreviations: SD, standard deviation 


\section{Table 2}

Number $(\%)$ of participants with events, by treatment assignment and by time period.

\begin{tabular}{|l|c|c|}
\hline & Usual Care Group (n=77) & Intervention Group (n=80) \\
\hline During 6-month treatment period & & \\
\hline Death or major adverse cardiac event & $11(14 \%)$ & $3(4 \%)$ \\
\hline Death & $1(1 \%)$ & $0(0 \%)$ \\
\hline Major adverse cardiac event & $11(14 \%)$ & $3(4 \%)$ \\
\hline During 12-month observational follow-up & & $11(14 \%)$ \\
\hline Death or major adverse cardiac event & $3(4 \%)$ & $2(3 \%)$ \\
\hline Death & $1(1 \%)$ & $9(11 \%)$ \\
\hline Major adverse cardiac event & $2(3 \%)$ & \\
\hline
\end{tabular}

Major adverse cardiac event is defined as hospitalization for myocardial infarction / unstable angina. 